\title{
Favorable attitudes toward testing for chromosomal abnormalities via analysis of fetal cells in maternal blood
}

\author{
Suzanne T. Zamerowski, $P h D^{1}$, Mark A. Lumley, $P h D^{2}$, Raoul A. Arreola, PhD ${ }^{3}$, Kimberly Dukes, $M A^{4}$,
} and Lisa Sullivan, $P h D^{5}$

\begin{abstract}
Purpose: The NICHD Fetal Cell Isolation Study (NIFTY) was a multicentered project to isolate fetal cells from maternal blood to detect fetal chromosomal abnormalities. The project included a psychosocial component, which is the basis of this article. We examined the attitudes of high-risk pregnant women toward the availability of a maternal blood test to identify fetal chromosomal abnormalities, how women would respond to hypothetical normal and abnormal maternal blood testing results, and the factors associated with a woman's preference to have an invasive procedure in response to a normal maternal blood test. Methods: High-risk pregnant women $(N=854)$ planning to have prenatal diagnostic invasive testing (amniocentesis or chorionic villus sampling) completed a survey. Results: The women highly favored maternal blood testing. Almost all women would seek invasive testing after an abnormal blood test. Only half of the women would seek invasive testing after a normal blood test; these women were older, more willing to terminate their pregnancy, and valued the increased accuracy of invasive testing more highly than women who would not have invasive testing after a normal maternal blood test. Conclusions: Women having invasive diagnostic testing welcome a noninvasive procedure that uses fetal cells in maternal blood, and its availability would decrease invasive testing by approximately $50 \%$. Research needs to examine the attitudes and anticipated responses of other risk groups as well as the effects of information about maternal blood test sensitivity and specificity on attitudes and responses. Genetics in Medicine, 2001:3(4):301-309.
\end{abstract}

Key Words: prenatal diagnosis, maternal blood analysis, serum screening, fetal cells, attitudes

Prenatal testing is creating a changing paradigm in antenatal care. Couples now have the option of fetal risk assessment, a stepwise approach to prenatal diagnosis, and an opportunity to make informed choices regarding pregnancy outcome. Advancing technology has contributed to the increased use of prenatal tests. One goal of Healthy People 2000 has been to increase to at least $90 \%$ the proportion of women in prenatal care who are offered screening and counseling regarding prenatal detection of fetal anomalies. ${ }^{1}$

Efforts have been under way to develop and improve the accuracy of first-trimester detection of fetal chromosomal abnormalities. For example, the National Institute of Child Health and Human Development has sponsored the Fetal Cell Isolation Study (NIFTY). This study sought to evaluate the possibility of isolating fetal erythrocytes from maternal blood, analyzing fetal chromosomal DNA to determine the fetal karyotype, and comparing these results with those ob-

\footnotetext{
From ${ }^{1}$ Villanova University, Philadelphia, Pennsylvania; ${ }^{2}$ Wayne State University, Detroit, Michigan; ${ }^{3}$ University of Tennessee Health Science Center; ${ }^{4}$ DM-STAT, Inc., Boston, Massachusetts; ${ }^{5}$ Boston University, Boston, Massachusetts.

Suzanne Zamerowski, PhD, Villanova University, Villanova, Pennsylvania 19085.

Received: March 7, 2001.

Accepted: April 27, 2001.
}

tained with traditional "gold standard" invasive diagnostic techniques (amniocentesis or chorionic villus sampling, CVS). The goal of the study was to investigate whether the "maternal blood test" would yield a prenatal diagnostic test with sensitivity and specificity for detecting and analyzing fetal chromosomal anomalies that is comparable to that of the invasive diagnostic tests. Thus, in contrast to various maternal serum-screening tests (e.g., alpha fetoprotein [AFP], human chorionic ganadotropin [hCG], unconjugated estriol [uE3]) that provide only information about risk, it was hoped that the fetal cell isolation technique would ultimately have the potential of replacing invasive diagnostic testing.

A concurrent goal of the NIFTY study was to examine the psychosocial implications attendant to the development and use of this new maternal blood test. We believe that an examination of patient's attitudes and anticipated responses should be conducted concurrently with the development of the technologic aspects of testing. Traditionally, examination of psychosocial issues related to genetic testing has been deferred until after clinical adoption of the technology, at which point unanticipated questions and concerns often emerge. This approach has contributed to poor utilization of genetic testing and errors of omission in clinical application. ${ }^{2,3}$ 
As advances in prenatal genetic testing continue, first trimester serum screening may become a standardized component of prenatal care. This approach holds promise as a population screening tool to assess the risk for fetal chromosomal anomalies, potentially replacing the assignment of risk based on age, history, and other factors. Yet, relatively little is known about women's desires for such testing, how they would respond to normal or abnormal test results, and the sociodemographic and attitudinal factors that might explain women's responses to test results. ${ }^{4,5}$

Previously, in the related literature on second-trimester maternal serum alpha fetoprotein screening (MSAFP), researchers have examined women's attitudes and factors that predict responses. Based on a comprehensive literature review, Jorgensen ${ }^{6,7}$ proposed a conceptual model of the predictors of women's attitudes toward prenatal screening and diagnosis. This model includes personal factors (e.g., ethics, religion, age, anxiety, knowledge, prior experiences, financial status), societal factors (e.g., norms, media attention), attitudes of partners and other pregnant women, and the availability of health services. Other researchers have noted these factors and have added attitudes toward and history of pregnancy termination $^{8-12}$ as important predictors. Insufficient knowledge of test characteristics and incorrect risk perception have also been noted as leading to misunderstandings and subsequent psychological adjustment difficulties after MSAFP testing. ${ }^{13-19}$

The availability of prenatal maternal blood testing can present difficult choices-to participate or not, to pursue further invasive diagnostic testing if indicated, and whether to terminate the pregnancy if an abnormality is identified. It is important to examine women's attitudes about such procedures as well as the processes that women use in making their decisions. Women's attitudes often differ from those of their providers and the researchers who develop the technology, and a clearer understanding of women's opinions toward prenatal maternal blood testing will enable professionals to better guide and counsel women when and if this technology becomes widely available.

For this study, we examined women who were planning on having invasive prenatal diagnosis and who were relatively familiar with the testing options offered to high-risk women. We used this population principally because these women were also invited to participate in the technical part of NIFTY (comparing maternal blood fetal cell isolation and analysis with the results of invasive diagnostic testing), but it is also the case that high-risk individuals such as these women have personal experiences that are highly significant for creating and influencing public opinion..$^{20}$ In this study, we addressed the following research questions:

1. What are the attitudes of women who are considering having an invasive diagnostic procedure toward the availability of maternal blood testing?

2. Given the availability of a noninvasive diagnostic maternal blood test, would women anticipate feeling pressured to have this test?
3. What impact would normal or abnormal maternal blood test results have on women's decisions about whether to proceed to have invasive diagnostic testing?

4. To what degree is the decision to pursue further invasive testing after a normal blood test associated with demographic and obstetrical history characteristics, the perceived benefits of maternal blood testing versus invasive testing, the mother's concern about fetal health, and her attitudes toward pregnancy termination?

\section{METHODS}

\section{Patients}

A sample of high-risk pregnant women was recruited from reproductive genetics clinics at four metropolitan areas in the United States between 1998 and 2000. Pregnant women were eligible to participate if they met these inclusion criteria: at least 16 years of age; an assigned gestational age between 10 weeks, 0 days, and 20 weeks, 6 days; and considered high risk for fetal aneuploidy based upon advanced maternal age, fetal sonographic abnormality, prior history of fetal aneuploidy, or abnormal maternal screen. All of the women were referred by primary obstetricians and were considering having an invasive diagnostic test (amniocentesis or CVS).

\section{Procedures}

Women were asked to participate in the survey study when they presented to a clinic for genetic counseling and invasive testing. Genetic counselors or other members of the clinic invited the women to participate in the NIFTY study. The survey part of the NIFTY study was presented to patients and conducted independently of the technical part (fetal cell isolation from maternal blood), and some women who completed the survey chose not to participate in the technical part. Patients provided written informed consent to complete the survey, which had been approved by each site's institutional review board. Patients typically completed the survey after receiving genetic counseling, and all patients completed the survey before being informed of the results of their invasive testing.

\section{Survey}

We constructed a survey that inquired about demographics and obstetrical history, and that also contained 35 items measuring attitudes toward various aspects of prenatal testing. Response options to these items consisted of a 4-point scale of agreement $(1=$ disagree strongly, $2=$ disagree, $3=$ agree, $4=$ agree strongly $)$.

Some of the 35 items were examined individually, whereas other items were grouped into scales. (These single items as well as items in the scales are presented in Tables 3 and 4.) The single items assessed interest in and perceived pressure to have maternal blood testing as well as women's anticipated responses to normal or abnormal maternal blood testing. We also created multi-item scales to assess four attitude constructs. Scales were created by taking the mean of the items hypothesized to measure the construct, after reversing item response options, as needed, so that all items were scored in the same direction. 


\section{Perceived advantages of blood test and invasive test}

To ensure that all women had the same basic information about both invasive testing and maternal blood testing, we embedded in the survey brief descriptions of both of these approaches, along with their advantages and disadvantages. Invasive tests were described as follows: "The most commonly used prenatal tests are amniocentesis and chorionic villus sampling (CVS). These tests are invasive. That is, they involve inserting a needle or other instrument into your body to take a small sample of fluid or cells from around the fetus. They may also cause you some discomfort and have a small chance of causing a miscarriage. In addition, it takes up to 14 days to get the results back from these tests. However, these tests look for a number of chromosomal abnormalities and are highly accurate. They can tell with great confidence whether your fetus has a chromosomal abnormality."

The maternal blood test was described as follows: "A new prenatal testing procedure is being developed that will involve only drawing a sample of blood from your arm. This test will be relatively painless to administer. Although this test is safe, it may not be as accurate as the invasive tests such as amniocentesis or CVS."

The survey presented six items dealing with these two prenatal tests - three items pertaining to advantages of the maternal blood test, and three items pertaining to advantages of invasive testing. Women were asked to rate how much they would prefer the given test because of the specific advantage listed. Because the three reasons for preferring each approach were highly correlated with one another, responses to each set of three items were averaged to form two composite scales: "Advantages of Blood Test" and "Advantages of Invasive Test." Higher scores indicate greater perceived advantages of each approach.

\section{Willingness to terminate pregnancy}

Women were asked a series of six questions regarding the conditions under which they would terminate a pregnancy. We created a "Willingness to Terminate" scale by averaging these items. Higher scores on this scale indicate that the respondent was more willing to terminate her pregnancy.

\section{Concern for fetal status}

This construct was assessed by two items, which were averaged to yield the "Concern for Fetal Status" scale; higher scores indicate that the mother had more concern for the health of her fetus.

\section{Statistical analysis}

Our analyses included descriptive statistics on single items, and for in these items, we collapsed "agree strongly" and "agree" (and "disagree strongly" and "disagree") to yield a dichotomous index of agreement. We also calculated descriptive statistics (mean, standard deviation) on the four multi-item scales and calculated internal consistency reliabilities using Cronbach's alpha. Our primary analysis compared two groups of women-those who reported that they would follow a normal maternal blood test with invasive testing, and those who would have no further testing. We compared these two groups on demographic, obstetrical history, and attitudinal characteristics, using two-sample $t$ tests or chi-square tests on continuous or dichotomous variables, respectively. Finally, we conducted a multiple logistic regression analysis to examine simultaneously all of the characteristics that differentiated these two groups of women. In this logistic regression, we considered all variables that were significant at $P<0.05$ in the bivariate analyses. All significance tests were two-sided (alpha $=0.05$ ), and analyses were conducted using SPSS version 9.0.

\section{RESULTS}

\section{Demographic and obstetrical history of the sample}

A total of 877 women were recruited into the study. Data from 23 women were excluded because they did not meet our criterion of completing at least $75 \%$ of the survey items, leaving a final sample of 854 women. Table 1 presents demographic data for the final sample. The sample represents a relatively educated, affluent, married, European American population of women, which is fairly typical of women seeking prenatal diagnostic testing. Table 2 presents obstetrical history. Of note, the median gestational age at the time of the study was 12 weeks. Almost half of the women had previous experience with invasive diagnostic testing, and about one-third had previously terminated a pregnancy.

\section{Interest in maternal blood testing and perceived pressure to have testing}

Table 3 presents the percentage of women in the sample who responded affirmatively (either "agree" or "agree strongly") to various single items from the survey. As can be seen, the vast majority of women reported that they generally wanted to have the new blood test. We also asked several items about perceived pressure to have the blood test, if the test were available today. A substantial minority reported that they would feel like they had no choice but to have the test, or have the test only if their doctor recommended it. Only a small number of women reported that they would experience pressure from the baby's father to have the test.

Patients strongly endorsed a model of stepwise testing. As shown in Table 3, the vast majority of women reported that they would prefer to take the blood test first to determine whether invasive testing would be necessary.

\section{Perceived advantages of maternal blood test or invasive test, attitudes toward pregnancy termination, and concern for fetal health}

Table 4 presents the sample mean and internal consistency measures for the four attitude scales that were created from the survey. This sample of women rated the advantages of the new blood test relatively highly. In particular, its specific advantages of eliminating the risk of fetal loss and obtaining test results earlier in pregnancy received strong support. In contrast, women in this sample rated somewhat less highly the advantages of invasive testing, although the increased accuracy of this 
Table 1

Demographic description of women in the sample

\begin{tabular}{|c|c|}
\hline & $\begin{array}{c}\% \text { of sample or mean }(\mathrm{SD}) \text {, range } \\
\qquad(N=854)\end{array}$ \\
\hline Maternal age (years) & $36.6(3.7) 16-49$ \\
\hline Paternal age (years) & $37.8(5.3) 18-60$ \\
\hline Education (years) & $16.4(2.4) 10-20$ \\
\hline \multicolumn{2}{|l|}{ Ethnicity } \\
\hline European American & $86.5 \%$ \\
\hline Hispanic & $4.1 \%$ \\
\hline African American & $3.8 \%$ \\
\hline Asian & $2.6 \%$ \\
\hline Middle East/Arab & $1.3 \%$ \\
\hline Multiracial & $1.0 \%$ \\
\hline Native American & $0.7 \%$ \\
\hline \multicolumn{2}{|l|}{ Employment status } \\
\hline Working full-time & $60.5 \%$ \\
\hline Working part-time & $21.9 \%$ \\
\hline Not employed outside of home & $17.5 \%$ \\
\hline Married & $93.6 \%$ \\
\hline \multicolumn{2}{|l|}{ Annual household income } \\
\hline$<\$ 15,000$ & $1.3 \%$ \\
\hline$\$ 15,000-29,999$ & $2.7 \%$ \\
\hline$\$ 30,000-49,999$ & $9.1 \%$ \\
\hline$\$ 50,000-99,999$ & $35.8 \%$ \\
\hline$\$ 100,000+$ & $40.0 \%$ \\
\hline Not provided & $11.1 \%$ \\
\hline \multicolumn{2}{|l|}{ Religion } \\
\hline Catholic & $36.2 \%$ \\
\hline Protestant & $24.6 \%$ \\
\hline Jewish & $17.3 \%$ \\
\hline Muslim & $0.5 \%$ \\
\hline Buddhist & $0.5 \%$ \\
\hline Other & $7.9 \%$ \\
\hline No preference & $11.3 \%$ \\
\hline
\end{tabular}

approach received the most favorable ratings of the three items in the scale.

With respect to the women's attitudes toward pregnancy termination, the women in this sample generally endorsed the possibility of termination. For example, $33.4 \%$ of the women reported that they would "terminate the pregnancy rather than give birth to a baby that was abnormal in any way," whereas, only $7.7 \%$ of the women reported that they would not terminate their pregnancy "no matter how abnormal the fetus might be." Note that, as assessed with a separate single item, none of the women in this sample indicated they would terminate the
Table 2

Obstetrical history of women in the sample

\begin{tabular}{lc}
\hline \multicolumn{2}{l}{$\begin{array}{c}\text { \% of sample or mean (SD), range } \\
(N=854)\end{array}$} \\
\hline $\begin{array}{l}\text { Pregnancy duration (weeks) } \\
\text { Gravida }\end{array}$ \\
1 & $13.1(3.0) 6-24$ \\
2 & $27.6 \%$ \\
3 & $24.0 \%$ \\
4 & $14.6 \%$ \\
$5+$ & $16.4 \%$ \\
Live births & \\
0 & $33.1 \%$ \\
1 & $40.2 \%$ \\
2 & $19.5 \%$ \\
$3+$ & $7.1 \%$ \\
Prior invasive diagnostic test (\% yes) & $45.9 \%$ \\
Prior spontaneous abortion (miscarriages) & $39.6 \%$ \\
Prior pregnancy termination & $34.1 \%$ \\
Because of fetal anomaly & $6.2 \%$ \\
Because of maternal health concerns & $0.9 \%$ \\
Because of "other reasons" & $27.0 \%$ \\
\hline
\end{tabular}

pregnancy if the fetus were not the sex that they wanted. Thus, an initial concern that the availability of a noninvasive maternal blood testing might contribute to pregnancy termination based on preferred fetal gender was unfounded here.

Regarding women's concern about fetal health status, the sample showed a slight tendency to disagree, suggesting somewhat low levels of concern or worry for the sample as a whole. As an example, $71 \%$ of the women agreed (or strongly agreed) with the item, "I am sure that my fetus is fine."

\section{Decisions made in response to maternal blood testing}

We asked all of the women how they would respond to two hypothetical scenarios in which maternal blood testing yielded opposite results. We first reminded the women that maternal blood testing might not detect chromosomal abnormalities as accurately as invasive tests. In the first scenario, patients were asked to consider a situation in which their maternal blood test yielded findings that were "abnormal." As shown in Table 3, in this case, the vast majority of the women reported that they would have an invasive test to be sure of the results. In the second scenario, patients were asked to consider a situation in which their maternal blood test results were "normal." In this case, slightly more than half of these women reported that they would have no further tests done, whereas the rest of the women reported that they would have a follow-up invasive test just to be certain of the results. 


\section{Factors associated with a woman's plan to have an invasive test in response to a normal maternal blood test}

Because almost all of the women reported that they would respond to an abnormal blood test with follow-up invasive testing, we did not examine factors associated with women's responses to this scenario. In contrast, the sample was nearly evenly divided in their response to a normal blood test; thus, we sought to determine the factors associated with women's responses to this situation. Table 5 presents data for 12 characteristics on two groups of women: (1) those who indicated they would follow up a normal maternal blood test with invasive testing; and (2) those who indicated they would have no further testing. As shown in Table 5, eight characteristics were significantly different between the two groups. Demographically and obstetrically, women who would respond to a normal maternal blood test with invasive testing were older, had been pregnant for slightly less time, were more likely to have had invasive testing previously, and were more likely to have had a pregnancy termination. Attitudinally, such women valued the advantages of invasive testing more highly, valued the advantages of maternal blood testing less highly, were more willing to terminate a pregnancy, and had greater concern for fetal health status.

Because many of these characteristics were related, we conducted a multiple logistic regression with simultaneous entry, relating the mother's group status (whether or not she would pursue follow-up invasive testing after a normal blood test) to all eight of the significant characteristics as a set. This model yielded five variables that were simultaneously significant correlates of the mother's group status. The model was then pared to include only these five significant variables: the mother's age $(P=0.03)$, a history of pregnancy termination $(P=0.037)$, a willingness to terminate $(P=0.019)$, advantages of invasive testing $(P<0.0001)$, and advantages of blood testing $(P=$ 0.038 ). Specifically, a woman was 7.5 times more likely to pursue an invasive test after a normal blood test if she agreed with a preference for the advantages of invasive testing as opposed to disagreeing (95\% Wald CI: 5.3, 10.8), 1.7 times more likely if she were 40 versus 30 years old (CI: 1.1, 2.6), 1.5 times more likely if she had previously terminated a pregnancy (CI: 1.0, 2.1), 1.4 times more likely if she were willing to terminate her pregnancy (CI: 1.1, 1.8), and 1.4 times more likely if she did not prefer the advantages of maternal blood testing (CI: 1.0, 1.9). Overall, the final model of five variables correctly classified $80.1 \%$ of cases $(c=0.801)$.

\section{CONCLUSION}

These results indicate that pregnant women planning to have an invasive prenatal diagnostic test welcomed the opportunity for a diagnostic test to detect fetal chromosomal anomalies from fetal cells isolated from maternal blood. They strongly endorsed the advantages of this blood test, such as no fetal risk from the procedure, earlier availability of results, and reduced pain and discomfort. At the same time, these women
Table 3

Percentage of women who agreed ("strongly agree" or "agree") with various single items in the survey

\begin{tabular}{lc}
\hline Survey item & Percent agreeing \\
\hline "Would generally want to have this new blood test" & 97.3 \\
"Would feel like I had no choice but to have the blood test & 28.5 \\
done" & \\
"Have the test done only if my doctor recommended it" & 28.8 \\
"Would feel pressured by the baby's father to have the test \\
$\begin{array}{l}\text { done" } \\
\text { "Would prefer to take the blood test first so that I could } \\
\text { determine whether amniocentesis or chorionic villus } \\
\text { sampling would be necessary" }\end{array}$ \\
$\begin{array}{l}\text { "Would have invasive diagnostic test if the maternal blood } \\
\text { test was abnormal" }\end{array}$ \\
$\begin{array}{l}\text { "Would have no further testing if maternal blood test was } \\
\text { normal" }\end{array}$ \\
\hline
\end{tabular}

recognized the advantages of invasive testing, primarily the increased accuracy of the test and greater confidence in the results.

It was clear that women prefer to approach prenatal testing in a stepwise manner. The vast majority of participants favored an initial maternal blood test before making decisions about subsequent invasive testing. This preference for a potential noninvasive diagnostic test parallels findings from studies that found a preference for initial serum screening. For example, Kornman et al. ${ }^{4}$ examined women's attitudes regarding the option of first trimester serum screening among 158 low-risk woman who were having MSAFP and 96 high-risk woman having CVS. Seventy-six percent of the low-risk women indicated a preference for the earlier screening as did $44 \%$ of highrisk women. Easier termination and reassurance were the reasons given for the first-trimester preference. For similar reasons, other researchers have also found that a substantial percentage of women prefer prenatal testing to be conducted in the first trimester. ${ }^{21-24}$ Earlier studies regarding second-trimester MSAFP testing found that women would prefer serum screening, because it would allow a stepwise approach to prenatal diagnosis, despite the limited time frame for decision-making. ${ }^{25-27}$

Almost all of the women reported that they would have subsequent invasive testing if the maternal blood test returned an abnormal result. However, there were substantial differences in how women would respond if the initial blood test returned normal. Approximately half of the women said that they would have no further testing, whereas the other half reported that they would have invasive diagnostic testing anyway, to confirm the negative maternal blood test. Compared with women who would stop their evaluation after a normal maternal blood test, women who would proceed to have invasive testing were more likely to perceive their fetus at genetic risk, placed more value on the increased accuracy of invasive testing, and were more willing to terminate their pregnancies. These results support 
Table 4

Descriptive statistics (mean, SD) and internal consistency (Cronbach's alpha) for the four attitude scales

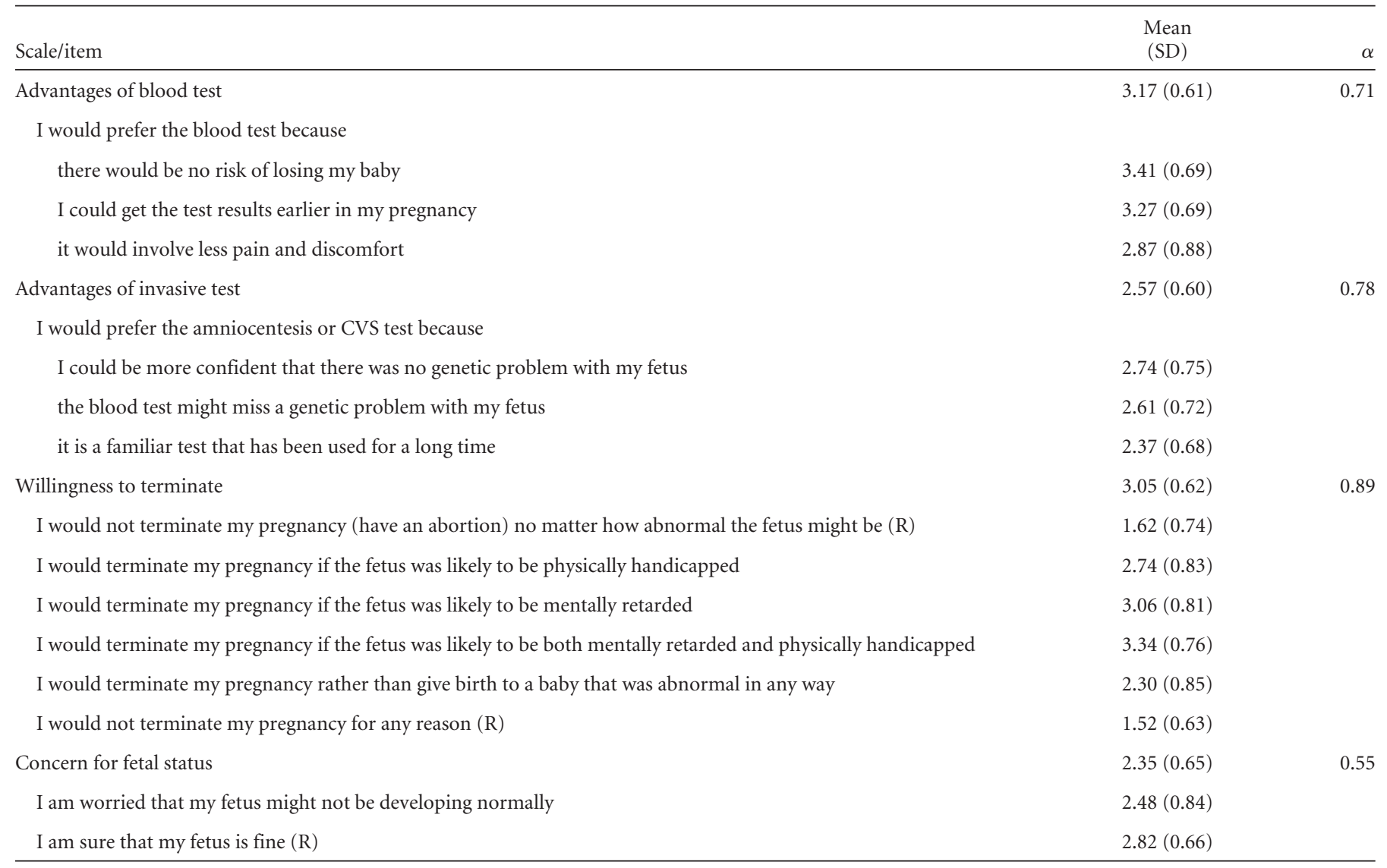

Note: Each scale is the average of items that were answered on a 4 -point scale, where $1=$ disagree strongly, $2=$ disagree, $3=$ agree, $4=$ agree strongly. $(\mathrm{R})$ denotes a reverse-scored item.

findings that a woman's risk perception, reproductive history, and attitudes toward abortion are strong predictors of their attitudes and course of action regarding prenatal testing. $9,12,16,28-31$ A woman's anxiety can influence her perceptions of risk and her attitudes about prenatal testing, and factors such as risk of fetal loss, test accuracy, attitudes toward termination, and timing of test results weigh heavily in a woman's decision regarding prenatal test choices and subsequent courses of action. ${ }^{32,33}$

Yet, one can ask why, although almost all of the women wanted to have the maternal blood first, approximately half of them would proceed to have invasive testing regardless of the results of the blood test? Why is their subsequent behavior not influenced by the blood test results? First, it should be remembered that the women in our sample were identified as high risk. The designation of being high risk creates increased anxiety for all women related to the concern for fetal health; however, in certain women, the response may be more intensified by individual factors (age, income, social support) and life events (acquaintance with a handicapped child, loss, grief, and an individual's trait anxiety). When a woman decides to undergo prenatal testing, the emotional distress can be even further intensified. Although prenatal testing is designed to pro- vide reassurance for some women, it intensifies anxieties and fears to the point that reassurance is difficult. ${ }^{34-36}$

Additionally, women in our sample were planning on having invasive testing anyway, so the high percentage of women who responded that they would follow a normal blood test with invasive testing might simply reflect the unique characteristics of our sample and setting. On the other hand, it is possible that the ease and risk-free nature of maternal blood testing makes it attractive as an initial approach but that the patients had not really thought about the consequences of positive or negative blood test results. When reminded that the blood test is "less accurate" than invasive testing, they sought the greater certainty of invasive testing.

This issue raises the problem of test accuracy and women's knowledge. Our survey described maternal blood testing as "less accurate" than invasive testing. We used this phrase primarily because, at the time our survey was designed, we did not know how the blood test would perform relative to invasive testing. But what does "less accurate" mean? This term fails to specify whether one is referring to sensitivity or specificity, positive or negative predictive value (which depend on base rates), or the range of genetic anomalies that can be detected. For example, traditional invasive testing identifies a broader range of chromo- 
Favorable attitudes toward maternal blood test

Table 5

Characteristics of women who would respond to a normal maternal blood test with further invasive testing versus no further testing

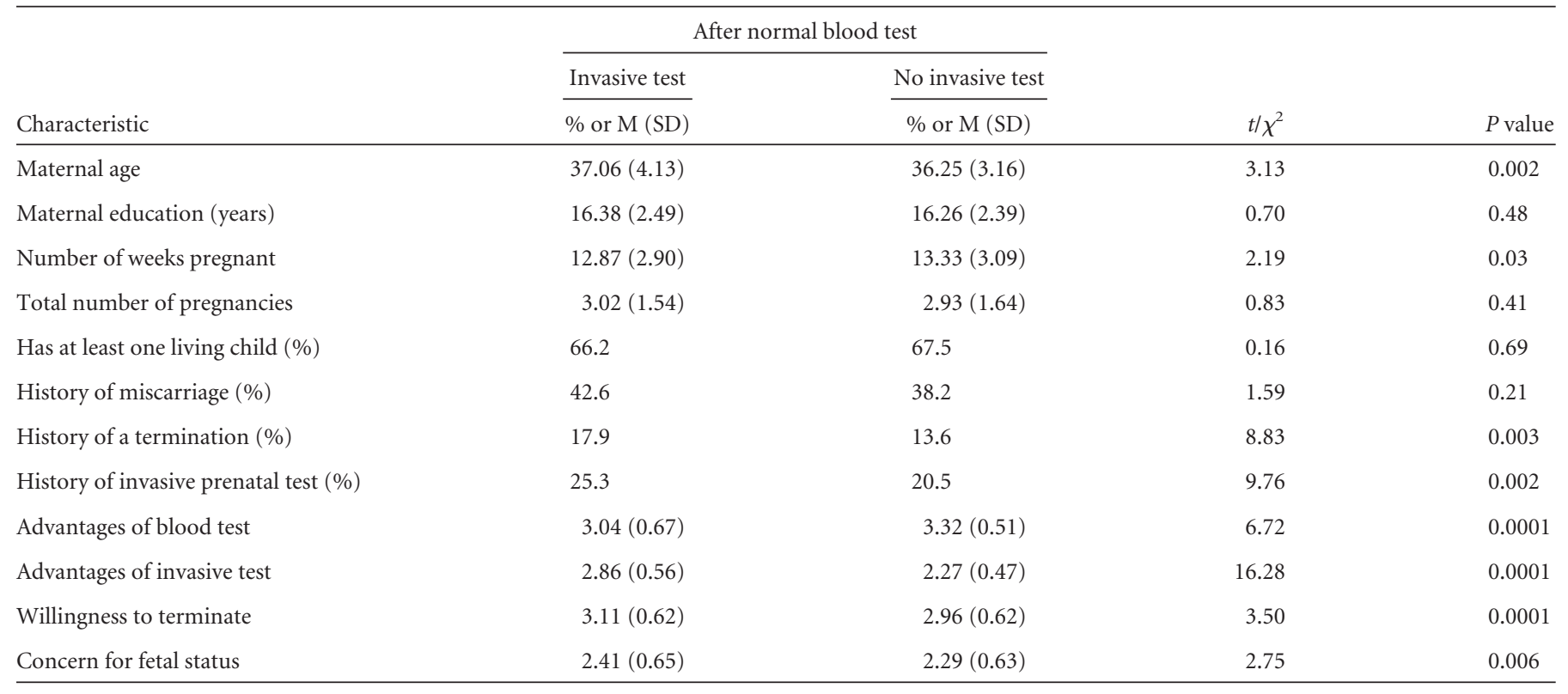

somal anomalies than do currently available techniques for analyzing chromosomes in fetal nucleated red blood cells. The concepts of probabilities, population risk of chromosomal disorders, and the difference between screening and follow-up diagnostic tests also are likely to be very confusing to many patients, as suggested by research in cognitive psychology. ${ }^{37}$

Yet, it appears that women's understanding of these test characteristics and principles is quite limited. Goel et al. ${ }^{19}$ studied women participating in prenatal screening and found that the majority did not have sufficient knowledge for truly informed consent, and inadequate knowledge contributed to misunderstandings regarding screening. Gekas et al. ${ }^{13}$ obtained similar results. Santalahti and colleagues ${ }^{14-16}$ studied women's knowledge and concluded that they need to know about test sensitivity, specificity, interpretation, and possible follow-up options before they can make truly informed decisions.

The potential availability of prenatal maternal blood testing raises the issue of whether this technology will become standard practice and routinely administered to all pregnant women, regardless of their desire for testing or their concerns about how to respond to the results. Studies on the freedom or voluntary nature of women's participation in prenatal screening have yielded conflicting results. On the one hand, Verp and Heckerling ${ }^{28}$ argued that every woman has a unique decisionmaking process that influences her choice and receptivity to prenatal screening, and Sjogren ${ }^{20}$ found that the most women sought autonomy regarding the use of reproductive technology and did not want authorities to make their decisions. On the other hand, Santalahti ${ }^{14}$ interviewed women in different phases of prenatal screening and asked them to describe their decision-making processes. More than $50 \%$ of these women reported that they considered their participation in prenatal screening as a "routine" or self-evident act, whereas only $25 \%$ actively decided whether to have prenatal screening. Marteau et al. ${ }^{38}$ reported that, despite prenatal guidelines, women often participate without being aware of possible outcomes of prenatal screening, and Porter and Macintyre ${ }^{39}$ concluded that women tend to assume that the system of prenatal care has been well thought out by the provider. Browner and Press ${ }^{10}$ found that pregnant women assume that technology is accurate and incontrovertible, so their use of it allows them to feel they are doing all they can to ensure a healthy fetus.

In our study, slightly less than a third of the women perceived pressure from society, the provider, or the father to have the maternal blood test. Although this is a minority of women in the sample, it is a substantial minority. Furthermore, this was found among women who were relatively highly educated and financially well-off - a group that one usually considers as more resistant to external pressures. Thus, it appears that many women are not making truly voluntary and informed decisions but are following the suggestions of their provider or expectations of society.

\section{Limitations and future research directions}

The sample of patients in the present study included only high-risk women who were planning on having invasive diagnostic testing. In addition, they were relatively educated, experienced regarding prenatal testing, and had favorable attitudes toward pregnancy termination. This limits our ability to generalize to other women. It is likely that the attitudes of these women differ from those of pregnant women not seeking invasive testing. Low-risk women, such as younger women or those without history of chromosomal abnormalities, may have less worry about the genetic status of the fetus. It is conceivable that they would make different choices not only about invasive testing, but also about initial blood testing as well, 
based on their lower perceived risk. There also is a population of women about whom less is known-high-risk women who decline invasive testing. Although their obstetricians may recommend invasive testing, some women decline, perhaps because of their religious beliefs, their views on pregnancy termination, their style of dealing with threatening information, or some other factors. It is likely that such women would respond differently to questions about the desirability of maternal blood testing or invasive testing. Therefore, future research should clarify the attitudes, motivations, and anticipated responses to maternal blood testing among women of low riskwho have the vast majority of pregnancies and who will likely be the ultimate target of advancing prenatal diagnostic technologies - as well as high-risk women who decline to have invasive testing.

In our society, women and men tend to assume that new medical technologies and their consequences have been adequately evaluated before implementation, and women do not usually refuse prenatal testing. We believe that the process of "fully informed, voluntary consent" is the central issue regarding decision-making and genetics. This is not only standard practice, but also ideal human care. Based on the literature and our study's findings, we suspect that many or most pregnant women have little understanding of the scientific and statistical issues involved in risk assessment and decision-making. Thus, future research should assess what women really understand about prenatal testing and develop interventions to increase their understanding so that they can make fully informed decisions. Also, studies are needed to clarify the nature and extent of the "voluntariness" of women's participation in these decisions. Research should examine both the internal and external sources of pressure (including interactions between patient, providers and family members), individual differences in the way that women respond to these influences, and how eventual decision-making interacts with the extent and accuracy of the patient's knowledge.

\section{Acknowledgments}

This research was supported by contracts N01-HD-4-3201, 3202, 3203, and 3204 from the National Institute of Child Health and Human Development. The authors acknowledge each of the study principal investigators: Diana Bianchi, MD, Sherman Elias, MD, Mark Evans, MD, Laird Jackson, MD, Joe Leigh Simpson, MD, Wolfgang Holzgreve, MD, Ronald Wapner, MD, and Sinuhe Hahn, PhD. The following individuals served as clinical coordinators for the study: Beth Berlin, MS, Eric Krivchenia, MS, Katie Leonard, MS, and Sue Moyer, MS. Personnel involved in data coordination, management, and analysis at DM-STAT included Amina Khan, MA, Patti Folan, Idania Ramirez, John Vidaver, MA, and Courtney Medeiros. The authors also thank Felix de la Cruz, MD, from the NIH for his support and guidance.

\section{References}

1. United States Department of Health, and Human Services. Healthy People 2000: National health promotion and disease prevention objectives (DHHS Publication No. [PHS] 91-50212). Washington, DC: US Government Printing Office, 1991.
2. Lashley F. The genetic revolution. Implications for nursing, 1997. Washington, DC: American Academy of Nursing.

3. Banta HD, Gelijns AC. The future of health care technology: implications of a system for early identification. World Health Stat Q 1994;47:140-148.

4. Kornman LH, Wortelboer MJM, Beekhuis JR, Morssink LP, Mantingh A. Women's opinions and the implications of first versus second-trimester screening for fetal Down's syndrome. Prenat Diagn 1997;17:1011-1018.

5. Zamerowski S, Lumley M, Arreola R, Dukes K, Kahn A, Sullivan L. The psychosocial impact on high-risk pregnant women of a non-invasive prenatal diagnostic test. Fetal Diagn Ther 1999;14:125-126.

6. Jorgensen FS. Attitudes to prenatal screening, diagnosis, and research among pregnant women who accept or decline an alpha-fetoprotein test. Prenat Diagn 1995;15: 419-429.

7. Jorgensen FS. User acceptability of an alpha-fetoprotein screening programme. Dan Med Bull 1995;42:100-105.

8. Lam YH, Tang MHY, Lee CP, Sin SY, Tang R, Wong HS, Wong SF. Acceptability of serum screening as an alternative to cytogenetic diagnosis of Down syndrome among women 35 years or older in Hong Kong. Prenat Diagn 2000;20:487-490.

9. Press N, Browner $\mathrm{CH}$. Characteristics of women who refuse an offer of prenatal diagnosis: data from the California maternal serum alpha fetoprotein blood test experience. Am J Med Genet 1998;78:433-445.

10. Browner $\mathrm{CH}$, Press N. The production of authoritative knowledge in American prenatal care. Med Anthropol Q 1996;16:141-156.

11. Rapp R. Sociocultural differences in the impact of amniocentesis. Fetal Diagn Ther 1993;(Suppl 1):90-96.

12. Rapp R. Amniocentesis in sociocultural perspective. J Genet Couns 1993;2:183-195.

13. Gekas J, Gondry J, Mazur S, Cesbron P, Thepot F. Informed consent to serum screening for Down syndrome: are women given adequate information? Prenat Diagn 1999;19:1-7.

14. Santalahti P, Hemminki E, Latikka AM, Ryynanen M. Women's decision making in prenatal screening. Soc Sci Med 1998;46:1067-1076.

15. Santalahti P, Aro AR, Hemminki E, Helenius H, Ryynanen N. On what grounds do women participate in screening? Prenat Diagn 1998;18:153-165.

16. Santalahti P, Latikka AM, Ryynanen M, Hemminki E. Women's experiences of prenatal serum screening. Birth 1996;23:101-107.

17. Glazier R, Goel V, Holzapfel S, Summers A, Pugh P, Yeung M. Written patient information about triple marker screening: a randomized controlled trial. Obstet Gynecol 1997;90:769-774.

18. Grewal GK, Moss HJ, Aitken DA, Bjornsson S, Cameron AD, Pell JP. Factors affecting women's knowledge of antenatal serum screening. Scott Med J 1997;42:111-113.

19. Goel V, Glazier R, Holzapeel S, Pugh P, Summers A. Evaluating patient's knowledge of maternal serum screening. Prenat Diagn 1996;16:425-430.

20. Sjogren B. Future use and development of prenatal diagnosis: consumers' attitudes. Prenat Diagn 1992;12:1-8.

21. Robinson GE, Garner DM, Olmsted MP, Shine J, Hutton EM, Crawford BM. Anxiety reduction after chorionic villus sampling and genetic amniocentesis. $\mathrm{Am} \mathrm{JOb-}$ stet Gynecol 1988;159:953-956.

22. Spencer JW, Cox DN. Emotional responses of pregnant women to chorionic villus sampling or amniocentesis. Am J Obstet Gynecol 1988;157:1155-1160.

23. Spencer JW, Cox DN. A comparison of chorionic villus sampling and amniocentesis: acceptability of procedure and maternal attachment to pregnancy. Obstet Gynecol 1987;72:714-717.

24. Lippman-Hand A, Perry TB, Mandell S, Cartier L. Chorionic villus sampling: women's attitudes. Am J Med Genet 1985;22:395-401.

25. Julian-Reynier C, Macquart-Moulin G, Moattit JP, Loundou A, Aurran Y, Chabal F, Ayme S. Attitudes of women of childbearing age towards prenatal diagnosis in South Eastern France. Prenat Diagn 1993;13:613-627.

26. Tymstra T. The imperative character of medical technology and the meaning of "anticipated decision regret." Int J Technol Assess Health Care 1991;7:509-516.

27. Roelofsen EEC, Kanerbeek LI, Tymstra RJ. Chances and choices: psychosocial consequences of maternal serum screening: a report from the Netherlands. J Reprod Infant Psychol 1993;11:4-47.

28. Verp MS, Heckerling PS. Use of decision analysis to evaluate patient's choices of diagnostic prenatal test. Am J Med Genet 1995;58:337-344.

29. Zamerowski ST. State-trait anxiety of women who have previously borne children with trisomy chromosome abnormalities and women of advanced maternal age prior to chorionic villus sampling [dissertation]. New York University, 1993.

30. Evers-Kiebooms G, Swerts A, Van Den Bergie H. Psychological aspects of amniocentesis: anxiety feelings in three different risk groups. Clin Genet 1988;33:196-206.

31. Beeson D, Golbus M. Anxiety engendered by amniocentesis. Birth Defects Orig Artic Ser 1979;15:191-197.

32. Weinstein ND, Klein WM. Resistance of personal risk perceptions to debiasing interventions. Health Psychol 1995;14:132-140.

33. Pauker SP, Kassirer JP. Decision analysis. N Engl J Med 1987;316:250-258. 
34. Kemp VH, Hatmaker DD. Stress and social support in high risk pregnancy. Res Nur Health 1989;12:331-336.

35. Robinson JO, Hibbard BM, Laurence KM. Anxiety during a crisis: emotional effects of screening for neural tube defects. J Psychosom Res 1984;28:163-168.

36. Blumberg B, Golbus MS, Hanson KH. The psychological sequelae of abortion performed for a genetic indication. Am J Obstet Gynecol 1975;122:799-809.
37. Tversky A, Kahneman D. The framing of decisions and the psychology of choice. Science 1981;211:453-458.

38. Marteau TM, Johnston M, Kidd J, Michie S, Cook R. Psychological models in predicting uptake of prenatal screening. Psychol Health 1992;6:13-22.

39. Porter M, Macintyre S. What is, must be best: a research note on conservative or deferential responses to antenatal care provision. Soc Sci Med 1984;19:1197-1200. 